\title{
Failure of combined costimulatory blockade in animal transplant model
}

To the editor-We read with interest the articles by Li et al. ${ }^{1}$ and Wells et al. ${ }^{2}$ in the November 1999 issue of Nature Medicine. They have made an impressive case for the fundamental role of activated T-cell apoptosis in the induction of long-term transplant tolerance. However, we were concerned about the lack of efficacy of combined co-stimulatory blockade (cytotoxic T-lymphocyte antigen 4 immunoglobulin (CTLA4-Ig) and mouse antibody against CD40 ligand (antiCD40L)) in preventing rejection of skin allografts in the fully major histocompatibility complex-mismatched mice, as reported by Li et al.

The most remarkable aspect about combined co-stimulatory blockade has been its universal success in inducing longterm graft acceptance in a variety of animal models ${ }^{3-6}$. Indeed Larsen and Pearson's original paper documented indefinite graft survival in skin grafts in the same strain combination (Balb/c to $\mathrm{C} 3 \mathrm{H} / \mathrm{He})$, using the same protocol ${ }^{4}$. Other studies have documented similar results in primate kidney grafts ${ }^{3}$, in bone marrow transplants ${ }^{6}$ and in xenotransplantation ${ }^{5}$. Thus, combined co-stimulatory blockade, rather than blockade of one co-stimulatory path alone, seems to work in even the most stringent transplant models. Yet Li et al. described the mean survival time for skin grafts that received both CTLA4Ig and the mouse anti-CD40L as only 15 days (range, 8-19), and not significantly different from that seen in controls. To our knowledge, this is the first report of a failure of this protocol to induce longterm skin graft survival.

It is not clear why the protocol failed, as a strain dependency or a difference in dosing regime does not seem to be the issue. The mechanisms for the failure need to be investigated. This may have considerable importance for upcoming human trials.

\footnotetext{
VIKAS R DHARNIDHARKA ${ }^{1}$,

KENNETH SCHOWENGERDT ${ }^{2} \&$

SUZANNE SKODA-SMITH ${ }^{3}$

${ }^{1}$ The Divisions of Nephrology, ${ }^{2}$ Cardiology and Immunology, ${ }^{3}$ Department of Pediatrics PO Box 100296

University of Florida College of Medicine Gainesville Florida 32610, USA

Email: vikasmd@peds.ufl.edu
}

Li and Strom reply-Dharnidharka and colleagues raise an important point. Costimulation blockade using anti-CD154 and CTLA4-Ig, albeit remarkably effective in many models, has not proven universally successful in producing permanent engraftment in allograft models. Although the cited work of Larsen and Pearson ${ }^{4}$ showed universal survival of skin allografts for 50 days, after further observation, only $50 \%$ of the recipients in that study experienced permanent engraftment (C. Larsen, personal communication). In skin graft models, subtle differences in the precise source of the skin graft, the source of CTLA4-Ig and differences in the $\mathrm{C} 3 \mathrm{H} / \mathrm{He}$ substrains are potentially very important in determining outcome. In fact, Larsen and Pearson have just shown in another strain combination that asialo $\mathrm{GM}^{+} \mathrm{CD} 8^{+} \mathrm{T}$ cells are essential in co-stimulation blockade-resistant mouse skin allograft rejection ${ }^{7}$. Several other groups have noted that $\mathrm{CD}^{+} \mathrm{T}$ cells are responsible for co-stimulation blockade resistant rejection in intesti$\mathrm{nal}^{8}$ and skin ${ }^{9}$ allograft models. And our own unpublished observations show that co-stimulation blockade is ineffective in blocking islet allograft rejection in a $\mathrm{CD}^{+} \mathrm{T}$-cell receptor transgenic model. We know of several other groups that have noted $\mathrm{CD}^{+} \mathrm{T}$-cell-dependent, co-stimulation blockade resistant allograft rejection. Recall that the expression of the co-stimulatory proteins differs from $\mathrm{CD}^{+}$to $\mathrm{CD}^{+} \mathrm{T}$ cells.

Larsen and Pearson have also failed to produce permanent engraftment in essentially the same subhuman primate kidney allograft model used by Kirk et al. (C. Larsen, personal communication). Larsen and Pearson use older monkeys than Kirk, and suspect that age related differences in the presence of memory cells in the older host may be important (C. Larsen, personal communication), as secondary immune responses are not as amenable to co-stimulation blockade as primary responses. A similar difference in the ability to tolerize young and old allograft responses with co-stimulation blockade has been noted in a rat model (H-D Volk, personal communication). And in an as-yet unpublished work, we found that co-stimulation blockade, even with the addition of donor specific transfusion, does not materially prolong survival of islet allografts placed into autoimmune non-obese diabetic mice.

In short, we believe that costimulation blockade based therapies have great promise for clinical application. Adjunctive therapies may well be required in many circumstances as memory $\mathrm{T}$ cells and, in some circumstances, naive $\mathrm{CD} 8^{+} \mathrm{T}$ cells are not ready tolerized after co-stimulation blockade. Although we do not advocate the addition of rapamycin ${ }^{2}$ as the only possible solution, we are convinced that a means to diminish the clone size of the responding alloreactive $\mathrm{T}$ cells through apoptosis or other measures is crucial to the success of co-stimulation blockade based and other potentially tolerizing therapies.

YongSheng Li \& TERRY B. STROM

Department of Medicine

Harvard Medical School

Research North, Rm 380

Division of Immunology

Beth Israel Deaconess Medical Center

P.O. Box 15707

Boston, Massachusetts 02215, USA

Email: tstrom@caregroup.harvard.edu

1. Li, Y. et al. Blocking both signal 1 and signal 2 of Tcell activation prevents apoptosis of alloreactive $T$ cells and induction of peripheral allograft tolerance. Naure Med. 5, 1298-302 2.(1999).

2. Wells, A.D. et al. Requirement for T-cell apoptosis in the induction of peripheral transplantation tolerance. Nature Med. 5, 1303-1307 (1999).

3. Kirk, A.D. et al. CTLA4-Ig and anti-CD40 ligand prevent renal allograft rejection in primates. Proc. Natl. Acad. Sci. USA 94, 8789-8794 (1997).

4. Larsen, C.P. et al. Long-term acceptance of skin and cardiac allografts after blocking CD40 and CD28 pathways. Nature 381, 434-438 (1996).

5. Elwood, E.T. et al. Prolonged acceptance of concordant and discordant xenografts with combined CD40 and CD28 pathway blockade. Transplantation 65, 1422-1428 (1998).

6. Wekerle, T. et al. Extrathymic T cell deletion and allogeneic stem cell engraftment induced with costimulatory blockade is followed by central T cell tolerance. J. Exp. Med. 187, 2037-2044 (1998).

7. Trambley, J. et al. Asialo $\mathrm{GM}^{+}{ }^{+} \mathrm{CD} 8^{+} \mathrm{T}$ cells play a critical role in costimulation blockade-resistant allograft rejection. J. Clin. Invest. 104, 1715-1722 (1999)

8. Newell K.A. et al. Blockade of the CD28/B7 costimulatory pathway inhibits intestinal allograft rejection mediated by $\mathrm{CD}^{+}$but not $\mathrm{CD}^{+} \mathrm{T}$ cells. J. Immunol. 163, 2358-2362 (1999)

9. Honey, K., Cobbold, S.P. \& Waldmann H. CD40 ligand blockade induces $\mathrm{CD} 4^{+} \mathrm{T}$ cell tolerance and linked suppression. J. Immunol. 163, 4805-4810 (1999). 